University of Nebraska - Lincoln

DigitalCommons@University of Nebraska - Lincoln

1992

\title{
Differential Toxicity of Enantiomers of Maackiain and Pisatin to Phytopathogenic Fungi
}

Leslie M. Delserone

University of Nebraska-Lincoln, Idelserone2@unl.edu

David E. Matthews

Cornell University

Hans D. VanEtten

Cornell University, vanetten@ag.arizona.edu

Follow this and additional works at: https://digitalcommons.unl.edu/libraryscience

Part of the Library and Information Science Commons

Delserone, Leslie M.; Matthews, David E.; and VanEtten, Hans D., "Differential Toxicity of Enantiomers of Maackiain and Pisatin to Phytopathogenic Fungi" (1992). Faculty Publications, UNL Libraries. 251.

https://digitalcommons.unl.edu/libraryscience/251

This Article is brought to you for free and open access by the Libraries at University of Nebraska-Lincoln at DigitalCommons@University of Nebraska - Lincoln. It has been accepted for inclusion in Faculty Publications, UNL Libraries by an authorized administrator of DigitalCommons@University of Nebraska - Lincoln. 


\title{
Differential Toxicity of Enantiomers of Maackiain and Pisatin to Phytopathogenic Fungi
}

\author{
Leslie M. Delserone, David E. Matthews, and Hans D. VanEtten \\ Department of Plant Pathology, Cornell University, Ithaca, NY 14853, USA \\ Present address for D. E. Matthews - Department of Plant Breeding and Biometry, Cornell University, Ithaca, NY 14853, USA \\ Corresponding author - H. D. VanEtten, Department of Plant Pathology, University of Arizona, Tucson, AZ 85721, USA
}

\begin{abstract}
Enantiomers of the isoflavonoid phytoalexins maackiain and pisatin were tested for toxicity to 36 fungal isolates representing 19 species. Nine of these species were pathogens of red clover, which synthesizes (-)maackiain; seven species were reported to be pathogenic on garden pea, which synthesizes predominantly (+)pisatin and minor amounts of $(-)$ maackiain. In general, non-host phytoalexins were more inhibitory than host phytoalexins to growth of these pathogens. In addition, the opposite enantiomer of the host phytoalexin was often more inhibitory to a pathogenic fungus than the normally-occurring enantiomer. There was little correlation between the tolerance of fungi to $(+)$ versus (-)pisatin and differential ability to metabolize the pisatin enantiomers. Nevertheless the data suggest that the production of non-host phytoalexins in transgenic plants might increase resistance of red clover and garden pea to some fungal pathogens.
\end{abstract}

Keywords: Pisum sativum, Sophora japonica, Trifolium pratense, Leguminosae, disease resistance, detoxification, tolerance, isoflavonoid, phytoalexin, pterocarpan

\section{Introduction}

Phytoalexins, antimicrobial compounds produced by plants in response to infection by microorganisms, are considered part of an active disease resistance mechanism [1]. Cruickshank et al. [2, 3] observed that (+)pisatin, the predominant phytoalexin synthesized by garden pea, generally was less toxic in vitro to fungal pathogens of pea than to non-pathogens, and he proposed that some pathogenic fungi circumvent disease resistance based on phytoalexin production by being tolerant of their host phytoalexins [4]. Exceptions exist to the general pattern described by Cruickshank $[5,6]$. For example, pisatin is quite toxic to Aphanomyces euteiches [7], but the organism colonizes pea tissue in which pisatin is present. However, in general, fungi are often found to be tolerant of host phytoalexins and sensitive to non-host phytoalexins, corroborating Cruickshank's observations [8]. In addition, experimental verification of Cruickshank's original proposal has been obtained in a few cases [9-11].

The potentially greater toxicity of non-host phytoalexins to pathogenic fungi might be exploited, at least in those cases where tolerance is required for pathogenicity, for disease control. The strategy would be to engineer plants to synthesize non-host phytoalexins, in addition to the normal complement of host compounds $[9,12]$. For this approach to be effective, the pathogens must be sensitive to the newly-synthesized non-host compounds as well as elicit their production.

In this study, 36 isolates representing 19 species of fungal pathogens and non-pathogens of red clover (Trifolium pratense L.) and garden pea (Pisum sativum L.), were assayed for growth inhibition by enantiomers of the isoflavonoid phytoalexins pisatin and maackiain (Figure 1). The fungi include members of the Ascomycetes, Basidiomycetes, and Deuteromycetes. Representatives of the Oomycetes were not included because their in vitro responses suggest that tolerance of host phytoalexins is not required for pathogenicity [6].

Red clover produces (-)maackiain, and pea produces predominantly $(+)$ pisatin and small amounts of $(-)$ maackiain [13]. Maackiain and pisatin were chosen for study because it appears possible, by the introduction of one or a few genes, to create transgenic plants which produce either enantiomers, or close analogues of these compounds (Figure 1) [9]. In addition, genetic and biochemical evidence indicates that tolerance of (-)maackiain and $(+)$ pisatin may be required by some fungi for pathogenicity to plants which produce these phytoalexins $[9,14)$.

The first objective of the present study was to test the toxicity of (-)maackiain and (+)pisatin as host phytoalexins. The responses of pathogens of plants that produce (-)maackiain and/or (+)pisatin were compared to the responses of fungi not pathogenic to plants which produce these compounds. Second, the enantiomers of each phytoalexin were compared for differential toxicity to the pathogens of a host which produces one of the enantiomers; specifically, the responses of red clover pathogens to (+) and (-)maackiain, and pea pathogens to enantiomers of both pisatin and maackiain, were evaluated. The third objective was to examine whether a host or nonhost compound was more easily metabolized, and whether the ability to metabolize a phytoalexin was related to tolerance of that compound. Pre- 


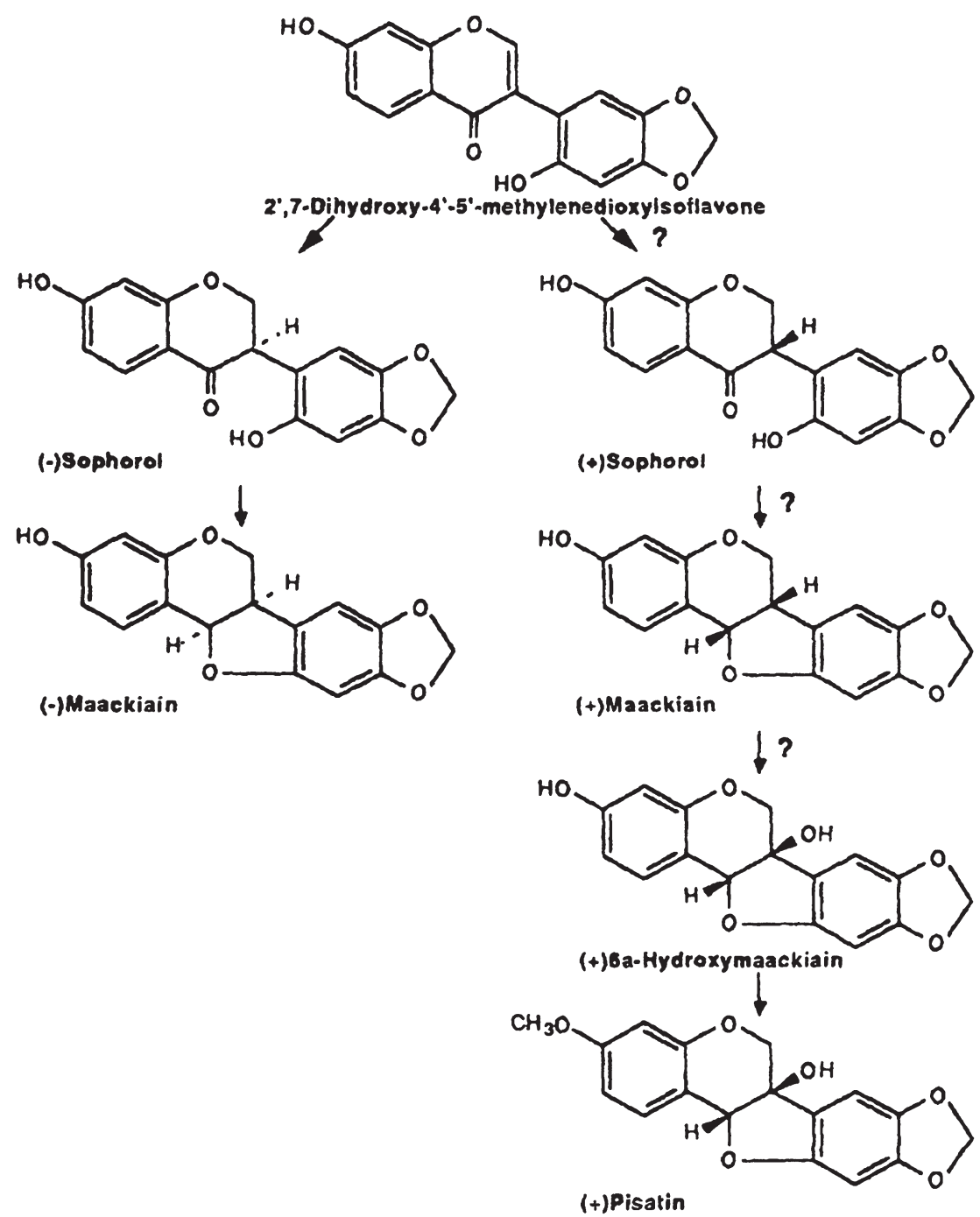

Figure 1. The proposed terminal steps in the biosynthetic pathways for (-)maackiain and for (+)pisatin [9]. Arrows marked with ? indicate conversions that have yet to be verified.

vious studies have indicated that detoxification is a tolerance mechanism for some fungi [9]. To address this objective, the abilities of selected fungi to metabolize $(+)$ and (-)pisatin were measured.

\section{Results}

\section{Toxicity of host phytoalexins}

The toxicity of a compound to a fungus was determined by recording the inhibition of radial mycelial growth of the fungus on agar medium, using a modification of a previously published assay [15]. An inhibition value of $40 \%$ was chosen as the value at which to classify a fungus as sensitive or tolerant to a phytoalexin. Based on previous studies with (+)pisatin and (-) maackiain in which this assay was used, isolates of Nectria haematococca mating population (MP) VI inhibited $\geq 40 \%$ were unable to metabolize pisatin or maackiain, and were low in virulence on pea [15-17] and chickpea
(Cicer arietinum L.) $[14,18]$. In cases where multiple isolates of a species were assayed, each isolate was evaluated individually in the comparison of the responses of pathogens with non-pathogens.

Fungi which infect red clover, Sophora japonica L. (Japanese pagoda tree), chickpea, or pea (Table 1) should encounter (-)maackiain as a host phytoalexin [13], while fungi isolated from Indigofera sp., corn (Zea mays L.) and bean (Phaseolus vulgaris L.) would not. (-)Maackiain was more toxic to the non-pathogens of (-)maackiain-producing plants than to the pathogens (Figure 2). Five of seven non-pathogens were inhibited more than $40 \%$, whereas only three of 29 pathogens were. Similarly, (+)pisatin was more toxic to fungi which do not colonize pea than to fungi which do (Figure 3). Among non-pathogens of pea, 17 of 23 were inhibited more than $40 \%$ by (+)pisatin, while only two of the 13 pathogens were. These two sensitive isolates (28 and 29) were isolated originally from pea but proved to be nonpathogenic when tested. 
Table 1. Species of fungi tested, their plant and geographic source, other relevant leguminous hosts, and the ability of selected isolates to metabolixe pisatin

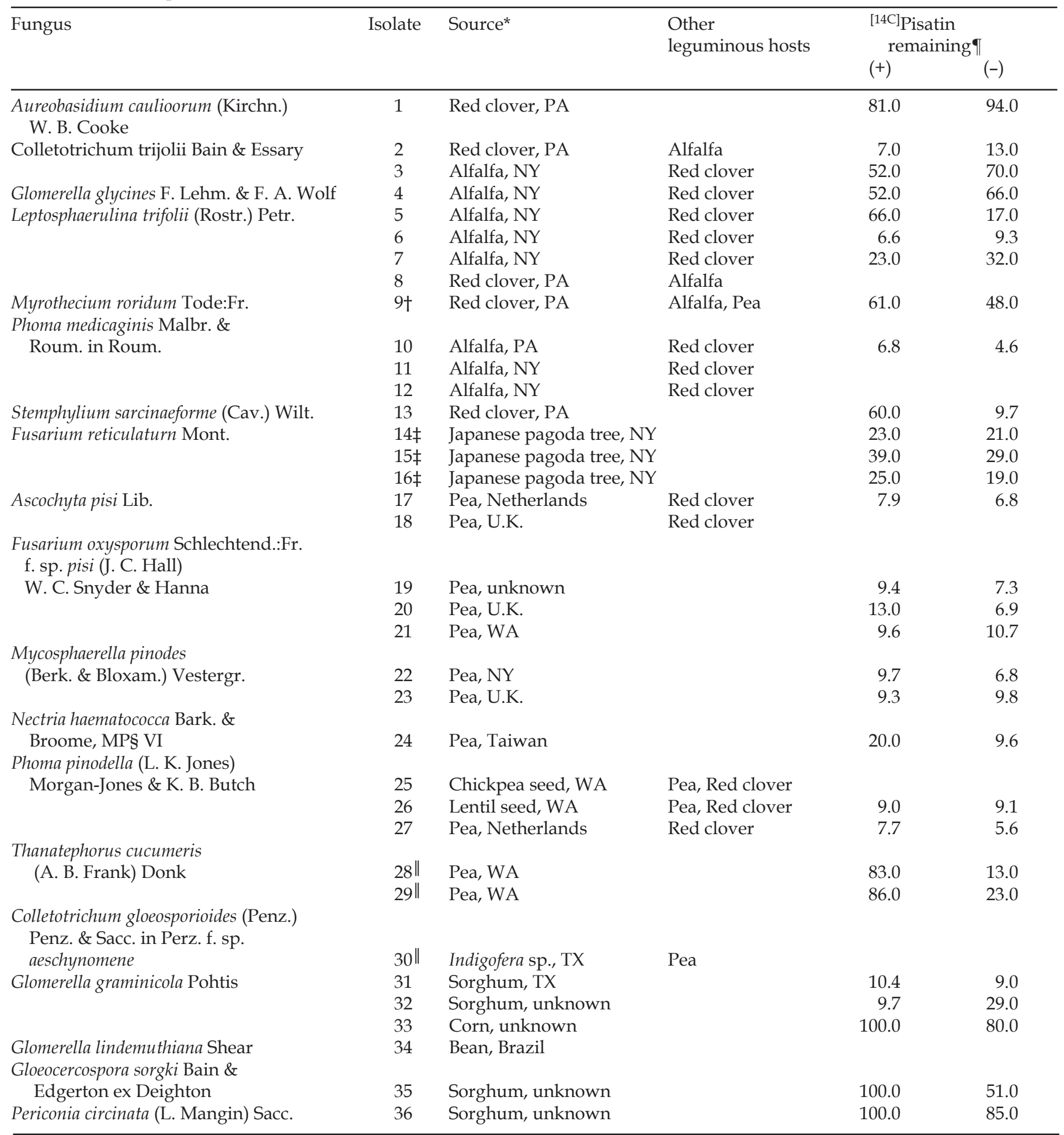

* Simple pterocarpanoid phytoalexins produced by the source plants [13]: red clover, (-)maackiain and (-)medicarpin; alfalfa, (-) medicarpin; Japanese pagoda tree, $(-)$ medicarpin, $(+)$ and (-)maackiain; pea, $(-)$ maackiiin, $(+)$ pisatin.

$\dagger$ Pathogenicity of this isolate on pea was not confirmed in the present study.

$\ddagger$ Pathogenicity of this isolate on Japanese pagoda tree was not confirmed in the present study.

$\S \mathrm{MP}=$ mating population.

|| This isolate was non-pathogenic on pea when tested in the present study.

II Percentage of $\left[{ }^{14} \mathrm{C}\right]$ pisatin $(0.1 \mathrm{mM})$ remaining after 7 days (mean of two experiments). 


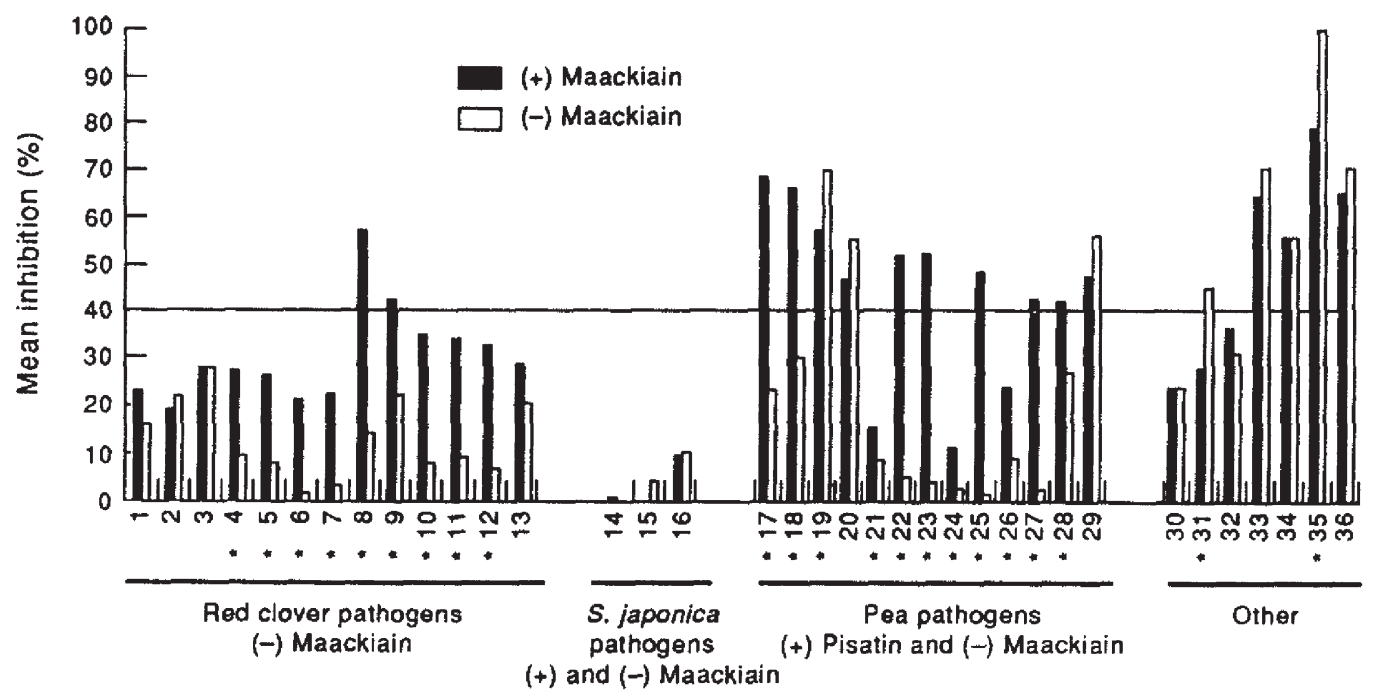

Isolate number

Figure 2. Mean inhibition of mycelial growth by enantiomers of maackiain. Values are expressed as a percentage relative to growth on control plates, and are the means of three to eight experimental means. Isolate numbers with asterisks indicate significant differences between enantiomers, determined in a one-tailed Mann-Whitney rank sum test $(P=0.05)$. Hosts of these fungi and the phytoalexins they produce are noted below the isolate numbers. "Other" refers to isolates not pathogenic on a plant producing either enantiomer of maackiain.

\section{Differential toxicity of enantiomers}

In comparisons of the toxicity of $(+)$ and (-)maackiain to pathogens of (-)maackiain-producing plants, 20 of 29 isolates reacted to the enantiomers differentially (Figure 2). In 19 cases, (+)maackiain was more toxic than (-)maackiain. The isolates of Fusarium reticulatum, which infect a plant that produces both enantiomers [19], were not significantly affected by either compound (Figure 2).

On the other hand, (-)pisatin does not appear to be generally more toxic than (+)pisatin, either to pea pathogens or to other fungi (Figure 3). Ten of the 13 pea pathogens were approximately equally tolerant to both enantiomers. Twelve of 13 pathogens of red clover were inhibited more than $40 \%$ by one or both of the enantiomers. Although eight of these isolates showed significant sensitivity to the pisatin enantiomers, differences in each direction were equally frequent. An exception was isolate 1 (Aureobasidium caulivorum), which was inhibited strongly by (-)pisatin and not at all by (+)pisatin. The pathogens of S. japonica were unaffected by either $(+)$ or $(-)$ pisatin.

\section{Metabolism of pisatin enantiomers}

The ability of 29 of the fungal isolates (representing 17 species) to metabolize pisatin was assayed by a modification of a previously published procedure [17]. The modification was a lower concentration of pisatin than that typically used in the assay; the lower concentration had been shown previously to be non-inhibitory to the fungal isolates. Isolates for which $\leq 80 \%$ of the pisatin remained were considered able to metabolize pisatin. All but six of the 29 isolates could metabolize both $(+)$ and (-)pisatin (Table 1). Of these six, all of which were non-pathogens of pea, three isolates $(1,33$, and 36$)$ could metabolize neither compound and the other three (28, 29 , and 35) could metabolize only (-)pisatin. Two isolates, 5 and 13, metabolized (-)pisatin more extensively than $(+)$ pisatin, but the rest metabolized both enantiomers approximately equally.

\section{Discussion}

$(-)$ Maackiain and (+)pisatin generally were more toxic to fungi which would not encounter these compounds in planta. The data agree with the pattern described by Cruickshank and others $[2,3,8,20,21]$. As they observed, the pattern is not an absolute one; for example, F. reticulatum was unaffected by either $(+)$ or $(-)$ pisatin.

$(+)$ Maackiain was generally more toxic than (-) maackiain to fungi which colonize red clover and pea. That is, in some cases alteration in the stereochemistry of a host phytoalexin produces a compound that is more toxic to a pathogen than the normally-occurring enantiomer, and suggests that plants engineered to produce $(+)$ maackiain might have increased resistance to some pathogens. This result differs from that of Sekido et al. [22]. In work with oryzalexins, phytoalexins synthesized by rice (Oryza sativa L.), the native (+) enantiomers were more inhibitory than the non-host (-) enantiomers to Magnaporthe grisea, the rice blast fungus.

Few of the pea pathogens were affected significantly by either enantiomer of pisatin. Based on this evidence, it appears that the production of (-)pisatin would not be a useful approach to increasing the resistance of pea to its pathogens. A large number of the fungi were able to metabolize the pisatin enantiomers, and there was little correlation between ability to metabolize an enantiomer and 


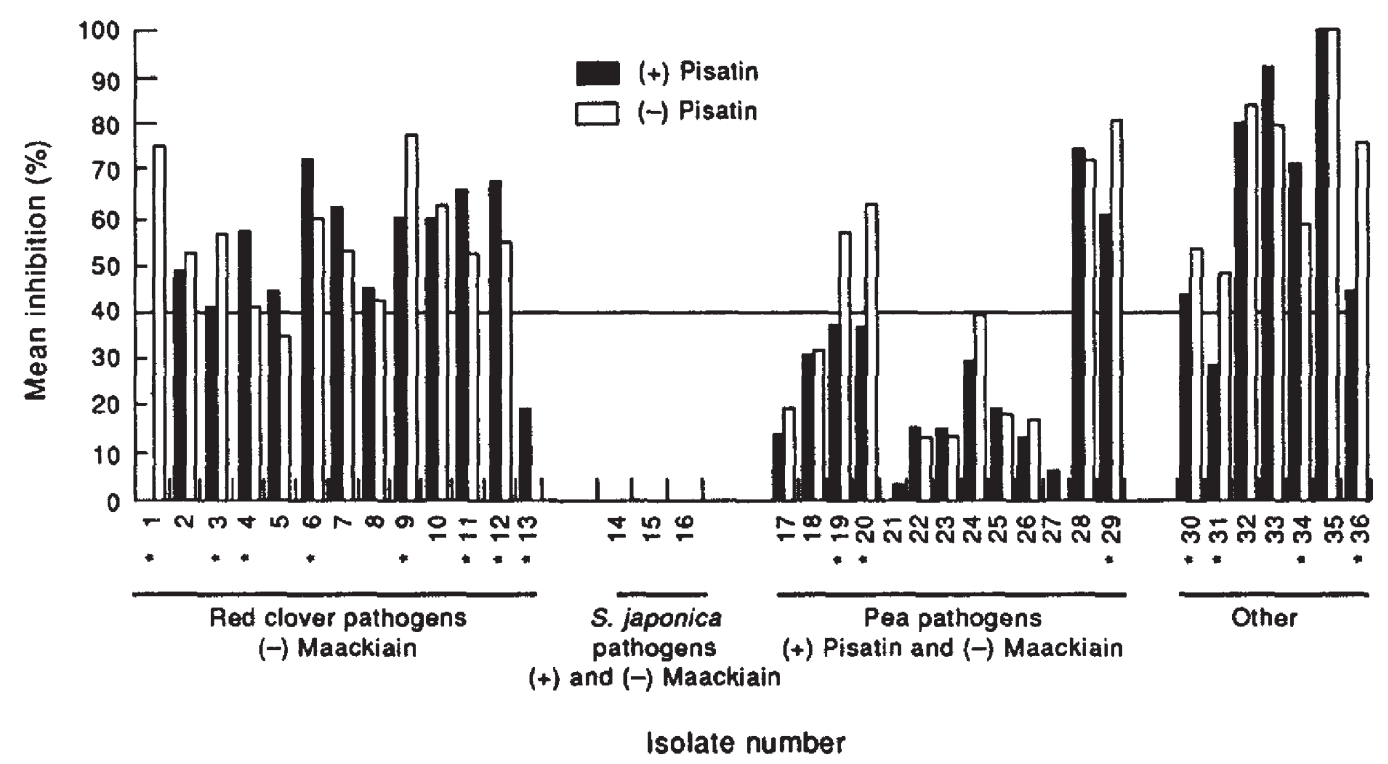

Figure 3. Mean inhibition of mycelial growth by enantiomers of pisatin. Values are expressed as a percentage relative to growth on control plates, and are the means of three to eight experimental means. Isolate numbers with asterisks indicate significant differences between enantiomers, determined in a one-tailed Mann-Whitney rank sum test $(P=0.05)$. Hosts of these fungi and the phytoalexins they produce are noted below the isolate numbers. "Other" refers to isolates not pathogenic on a plant producing either enantiomer of pisatin.

differential tolerance to it. For example, isolate 1 could not metabolize either enantiomer, yet demonstrated extreme differences in sensivitity to the compounds; isolates 28 and 29 metabolized only (-)pisatin, but had similar sensitivities to the compounds (Table 1, Figure 3). Demethylation of $(+)$ pisatin has been implicated as a key detoxification step for several fungal pathogens of pea, particularly N. haematococca MP VI [9], and the rate at which demethylation occurs may also be important for tolerance in this fungus [15]. Isolates of N. haematococca able to demethylate at moderate to rapid rates are tolerant of pisatin, while isolates with a slow rate are more sensitive to pisatin, as are those with no demethylating ability. In the present study, a noninhibitory concentration of pisatin was used for the metabolism assay, and the amount of pisatin remaining was measured after the fungus had ramified throughout the amended agar medium. The assay did not measure the rate of pisatin metabolism during exposure to an inhibitory concentration; rather, it was a stringent test of whether an organism had the capacity to degrade pisatin. Furthermore, tolerance mechanisms other than detoxification [10,23] may exist for some of the isolates used in the present study, such as differences in fungal membranes which might allow differential sensitivity to pisatin $[5,10]$. However, the results of the present study support the conclusions of other researchers that the ability to metabolize a phytoalexin is not always associated with tolerance to the compound or with the host range of the fungus $[6,9,10]$.

Linear growth of mycelia in vitro is not the only factor to consider in evaluating the potential toxicity of maackiain and pisatin to fungi $[5,10]$. There are many other factors involved in pathogenesis and the survival and reproduction of a fungus. For example, several red clover isolates, upon exposure to $(+)$ maackiain, failed to produce melanized mycelia and pycnidia, although both were present upon exposure to (-)maackiain (data not shown). Also, the bioassays did not test mixtures of phytoalexins, which occur naturally and might interact synergistically or antagonistically [10].

The information presented here is useful in observing trends which are potentially applicable in disease management. The evidence suggests that the presence of nonhost phytoalexins might improve the disease resistance of red clover and pea to some fungi. The biosynthetic pathways of the pterocarpan phytoalexins have been largely determined $[9,10]$, although some of the enzymes involved have yet to be characterized, and the corresponding genes have not been identified and isolated. As a result, the number of genes needed for the production of non-host phytoalexins in a given host is unknown. However, only a few genes would be required to engineer the terminal steps in the biosynthesis of a specific phytoalexin in a plant that already produces a related compound. For example, two steps would be required to engineer (-)pisatin biosynthesis in red clover, which normally produces (-)maackiain (Figure 1). Another possible tactic involves manipulation of the stereochemistry of the host phytoalexin, e.g. engineering red clover or pea to produce the non-host phytoalexin $(+)$ maackiain in addition to (-)maackiain (Figure 1). It is unknown whether fungal pathogens would eventually become tolerant of the nonhost compounds and, if so, how fast this resistance might develop within a pathogen population. It is now feasible, however, to engineer plants to synthesize non-host phytoalexins [12], and to test the effectiveness and stability of the disease resistance in these transgenic plants. 


\section{Experimental}

Pkytoalexins. The (-) and (+) enantiomers of maackiain were extracted from roots of red clover and S. japonica, respectively, and purified and crystallized as previously described [19]. (+)Pisatin was extracted from pea [24]. (-)Pisatin, as well as the two enantiomers of pisatin specifically radiolabelled at the 3-O-methyl position with ${ }^{14} \mathrm{C}$, were prepared by methylation of $(-)$ or $(+) 6 a-h y d r o x y m a a c k i a i n ~(6 a-H M)$ with methyl iodide [16]. (+)6a-HM was obtained by 6a-hydroxylation of (-) maackiain by N. haematococca MP VI isolated T-95 [19]. (+)6aHM was obtained by demethylation of $(+)$ pisatin by transformant III-202 of Aspergillus nidulans strain UCDI, as previously described [25]. Maackiain and pisatin were quantified using the molar extinction coefficients in $\mathrm{EtOH}, \lambda_{\max } \mathrm{nm}(\log \varepsilon): 310$ (3.899) for maackiain [26], and $\lambda_{\max } n m(\log \varepsilon)$ : 309 (3.86) for pisatin [27].

Cultures. The fungi were isolated from diseased portions of the source plants indicated in Table 1. Isolates were provided by the following individuals (isolate number in this study, followed by donor's designation, if any): G. C. Bergstrom (10, Pm866; 11, Pm872; 12, Pm873), J. E. Carroll (14-16) and Ping Wang (31-33, 35-36), all of Cornell University, USA M. Gerlagh, Institute for Plant Protection, Netherlands (17, E457; 27, 565); W. J. Kaiser, Washington State University, Pullman, USA (25, ATCC 58662; 26, ATCC 58660); J. Kraft, IAREC, Prosser, WA, USA (28, R23; 29, R36); K. T. Leath, USDA-ARS, The Pennsylvania State University, University Park, USA (1, 3; 2, H85; 4; 8,976; 9); P. Matthews, John Innes Institute, Norwich, U.K. (18, JIPD2; 20, JI2; 21, JI22; 23, JI29); R. J. Rodriguez, University of Califomia-Riverside, USA (34, BA10 [28]); T. E. Stasz, NY State Agricultural Experiment Station, Geneva, USA $(22$, CY); and G. Templeton, University of Arkansas, Fayetteville, USA (30). All fungi isolated from alfalfa and red clover were confirmed as pathogens of red clover by others. The isolates of F. reticulaturn were not tested for pathogenicity. All pea isolates used in this study were tested for pathogenicity on pea, as previously described [16, 29]; those that proved to be non-pathogenic are noted in Table 1. Isolates 30-36 were also tested and found to be non-pathogenic on pea. All isolates were maintained on V-8 agar medium slants [30] at $4^{\circ}$, except for the Fusarium sp., which were maintained as single-spored isolates on carnation leaf agar [31] at $4^{\circ}$.

Assay of sensitivity to phytoalexins. Inoculum cultures were grown on Martin's peptone-glucose agar (PGA) medium [32] for 2-3 days at $24 \pm 1^{\circ}$, in darkness. A 4 mm-diameter agar plug with mycelia was removed with a sterile cork borer from the advancing margin of the inoculum culture. The plug was placed mycelium-side down on the surface of $1.0 \mathrm{ml}$ PGA amended with $(+)$ or $(-)$ maackiain in EtOH or $(+)$ or $(-)$ pisatin in DMSO, in a plastic petri plate $(35 \times 10 \mathrm{~mm})$. Final cones of maackiain and pisatin were $55 \mu \mathrm{g} \mathrm{ml}^{-1}$ (ca $0.2 \mathrm{mM}$ ) and $161 \mu \mathrm{g} \mathrm{ml}^{-1}(0.5 \mathrm{mM})$, respectively. These concentrations of phytoalexins were near the maximum of solubility, given the volume of organic solvent used. Control plates contained PGA amended solely with EtOH $(0.5 \%)$ or DMSO $(1.0 \%)$. Plates containing unamended PGA were also inoculated to determine any growth inhibition due to the solvent, which was generally $10 \%$ or less. The assay plates were incubated at 24 $\pm 1^{\circ}$ in darkness, a temperature that allowed near-maximum growth rates for most of the fungi tested. The radius of the col- ony was measured to the nearest $0.5 \mathrm{~mm}$ for each plate at 12 to $24 \mathrm{hr}$ intervals, until mycelia grew to the edge of the solvent-amended control plates, or for a maximum of 10 days. Colony radius at this point was termed endpoint growth. The endpoint inhibition (EI) value was the colony radius (endpoint growth) in phytoalexin-amended PGA as a percentage of that in solvent-amended medium.

Growth curves often exhibited an initial period (up to 3 days) of slow growth (lag phase), followed by a linear phase of growth. In N. haematococca, pisatin has two different effects on the growth curves of pisatin-sensitive, low-virulence isolates: slowly demethylating isolates showed a prolonged lag phase, whereas non-demethylating isolates had a lower final linear growth rate (LGR) [15]. The EI value reflects both kinds of effects, and is the value reported as "inhibition" in Figures 2 and 3. Analysis of the growth curves generated in the present study indicated that inhibition was primarily due to LGR effects for most isolates. Since the LGR value was similar to the EI value for the isolates used in this study, the EI values only are reported.

The range of the means, both within and among experiments, was generally $20 \%$ or less. In each experiment, means of EI were calculated for the three replicate plates in each isolate-treatment combination. The means from three to eight experiments were used in the Mann-Whitney rank sum test [33]. Each isolate was tested for differentiai sensitivity to $(+)$ versus $(-)$ maackiain, and $(+)$ versus $(-)$ pisatin, in a one-tailed test $(P$ $=0.05)$.

Metabolism of pisatin. Plastic scintillation vials $(7 \mathrm{ml})$ containing $0.25 \mathrm{ml}$ and PGA amended with either $(-)$ or $(+)$ [3-Omethyl- $\left.{ }^{14} \mathrm{C}\right]$ pisatin $\left(1-2 \times 10^{5} \mathrm{dpm} \mu \mathrm{mol}^{-1} ; 31 \mu \mathrm{g} \mathrm{ml}^{-1}\right)$ were each inoculated with one $4 \mathrm{~mm}$ diameter agar plug with mycelia taken from the advancing edge of a 2-3-day-old inoculum culture, one vial for each isolate-enantiomer combination. The vials were incubated at $24 \pm 1^{\circ}$ in darkness for 7 days, by which time the mycelia in each vial had grown over the agar surface. Scintillation fluid $(4.5 \mathrm{ml}$ of $0.55 \%$ 2,5-diphenyloxazole in toluene) was added to each vial at the end of the incubation period. The remaining labelled (unaltered) pisatin was partitioned into the toluene phase [17], and was measured in a scintillation spectrometer. The percentage of $(-)$ or $(+)$ pisatin remaining was calculated for each isolate. The assay measured pisatin metabolism via an initial demethylation step. Removal of the 3-O-methyl group is the only first step known for fungal metabolism of pisatin [6], and was verified as such for isolates 10-12, 17-27, and 30-32 [Delserone, unpublished]. The metabolism experiment was conducted twice; the range of values between experiments was $10 \%$ or less.

Acknowledgments - Our thanks to Patty S. Matthews for excellent technical assistance, and to the individuals who provided isolates for this study. Special thanks to P. E. Nelson, Fusarium Research Center, The Pennsylvania State University, for the identification of the isolates of Fusarium reticulaturn. This research was supported in part by National Research Initiative Competitive Grant No. 87-CRCR-1-2510 from the Coooperative State Research Service and by USDA Food and Agricultural Sciences National Needs Graduate Fellowship Program Grant 84-GRAD-9-0039 (to L.M.D.). 


\section{References}

1. Paxton, J. D. (1981) Phytopathol. Z. 101, 106.

2. Cruickshank, I. A. M. (1962) Aust. J. Biol. Sci. 15, 147.

3. Cruickshank, I. A. M. and Perrin, D. R. (1963) Aust. J. Biol. Sci. 16, 111.

4. Cruickshank, I. A. M. (1965) Tagungsber. Deutsch. Akad. Lundwirtschaftswiss. Berlin 74, 313.

5. Smith, D. A. (1982) in Phytoalexins (Bailey, J. A., and Mansfield, J. W., eds), p. 218. Blackie, Glasgow.

6. VanEtten, H. D., Matthews, D. E., and Smith, D. A. (1982) in Phytoalexins (Bailey, J. A., and Mansfield, J. W., eds), p. 181. Blackie, Glasgow.

7. Pueppke, S. G. and VanEtten, H. D. (1976) Phytopathology 66, 1174.

8. Harborne, J. B., and Ingham, J. L. (1978) in Biochemical Aspects of Plant and Animal Coevolution (Harborne, J. B., ed.), p. 343. Academic Press, New York.

9. VanEtten, H. D., Matthews, D. E., and Matthews, P. S. (1989) Ann. Rev. Phytopathol. 21, 143.

10. Smith, D. A., and Banks, S. W. (1986) Phytochemistry 25, 979.

11. Desjardins, A. E., and Gardner, H. W. (1989) Mol. Plant-Microbe Interact. 2, 26.

12. Hain, R., Bieseler, B., Kindl, H., Schröder, G., and Stocker, R. (1990) Plant Mol. Biol. 15, 325.

13. Ingham, J. L. (1982) in Phytoalexins (Bailey, J. A., and Mansfield, J. W., eds), p. 21. Blackie, Glasgow.

14. Miao, V. P. W., and VanEtten, H. D. (1992) Appl. Env. Microbiol. 58, 809 .

15. Kistler, H. C., and VanEtten, H. D. (1984) J. Gen. Microbiol. 130, 2605.

16. VanEtten, H. D., Matthews, P. S., Tegtmeier, K. J., Dietert, M. F., and Stein, J. I. (1980) Physiol. Plant Pathol. 16, 257.
17. Mackintosh, S. F., Matthews, D. E., and VanEtten, H, D. (1989) Mol. Plant-Microbe Interact. 2, 354.

18. Lucy, M. C., Matthews, P. S., and VanEtten, H. D. (1988) Physiol. Mol. Plant Pathol. 33, 187.

19. VanEtten, H. D., Matthews, P. S., and Mercer, E. H. (1983) Phytochemistry 22, 2291.

20. Cruickshank, I. A. M., and Perrin, D. R. (1971) Phytopathol. Z. 70, 209.

21. Perrin, D. R., Biggs, D. R., and Cruickshank, I. A. M. (1974) Aust. J. Chem. 27, 1607.

22. Sekido, H., Kamada, K., Kodama, O., and Akatsuka, T. (1987) Agric. Biol. Chem. 51, 2017.

23. Denny, T. P., and VanEtten, H. D. (1983) J. Gen. Microbial. 129, 2893.

24. Sweigard, J., and VanEtten, H. D. (1987) Phytopathology 77, 771.

25. Preisig, C. L., Matthews, D. E., and VanEtten, H. D. (1989) Plant Physiol. 91, 559.

26. Pert-in, D. R., and Bottomley, W. (1962) J. Am. Chem. Soc. 84, 1919.

27. Cruickshank, I. A. M., and Perrin, D. R. (1961) Aust. J. Biol. Sci. 14, 336.

28. Rodriguez, R. J., and Yoder, O. C. (1987) Gene 54, 73.

29. Tegtmeier, K. J., and VanEtten, H. D. (1982) Phytopathology 12,608 .

30. Miller, P. M. (1955) Phytopathology 45, 461.

31. Fisher, N. L., Burgess, L. W., Toussoun, T. A., and Nelson, P. E. (1982) Phytopathology 72, 151.

32. Martin, J. P. (1950) Soil Sci. 69, 215.

33. Conover, W. J. (1980) Practical Nonparametric Statistics, 2nd ed., Wiley, New York. 